\title{
Statistics of close-packed dimers on fractal lattices
}

\author{
D Marčetić ${ }^{1}$, S Elezović-Hadžić ${ }^{2}$ and I Živić ${ }^{3}$ \\ ${ }^{1}$ Faculty of Natural Sciences and Mathematics, University of Banja Luka, \\ M. Stojanovića 2, Bosnia and Herzegovina \\ ${ }^{2}$ Faculty of Physics, University of Belgrade, P.O.Box 44, 11001 Belgrade, Serbia \\ ${ }^{3}$ Faculty of Science, University of Kragujevac, Radoja Domanovića 12, Kragujevac, \\ Serbia \\ E-mail: dusanka.marcetic-lekic@pmf.unibl.org, suki@ff.bg.ac.rs, \\ ivanz@kg.ac.rs
}

\begin{abstract}
We study the model of close-packed dimers on planar lattices belonging to the family of modified rectangular (MR) fractals, whose members are enumerated by an integer $p \geq 2$, as well as on the non-planar 4 -simplex fractal lattice. By applying an exact recurrence enumeration method, we determine the asymptotic forms for numbers of dimer coverings, and numerically calculate entropies per dimer in the thermodynamic limit, for a sequence of MR lattices with $2 \leq p \leq 8$ and for 4-simplex fractal. We find that the entropy per dimer on MR fractals is increasing function of the scaling parameter $p$, and for every considered $p$ it is smaller than the entropy per dimer of the same model on 4-simplex lattice. Obtained results are discussed and compared with the results obtained previously on some translationally invariant and fractal lattices.
\end{abstract}

Keywords: Close-packed dimer model; Fractals; Recursive enumeration; Entropy 


\section{Introduction}

A close-packed dimer model is a classical example of a lattice statistical mechanical model that can be solved exactly on regular planar lattices. It has emerged as a simplified version of the so called monomer-dimer model, that was introduced in 1937 by Fowler and Rushbroke [1] in their study of the liquid mixtures. Exact solution of the closepacked dimer model on the square lattice was given in the 60-ies by Kasteleyn [2, 3], and independently by Temperly and Fisher [4,5] and Fisher [6]. More recent solution on arbitrary planar bipartite graphs has been given by Kenyon et al [7]. Although introduced as a simple model for adsorption of diatomic molecules on crystal surfaces, connections with other models in physics and chemistry have been established since then. It has been shown that the close-packed dimer model is equivalent to the two dimensional Ising model [8], and correspondence with many quantum theoretical field theory models has been recognized [9-13. In graph theory the model is also referred to as perfect matchings and is closely related with other combinatorial objects such as domino tillings [14] and spaning trees [15, 16].

Besides the square lattice, the close-packed dimer model has also been studied on other translationally invariant lattices [17-19], on some graphs without translational symmetry, such as self-similar graphs [20,21], and particularly on the Sierpinski gasket lattice and its generalizations [22].

In this paper we analyze close-packed dimer model on the subset of planar fractal lattices that belong to the family of the modified rectangular (MR) lattice introduced by Dhar [23]. We also consider the model on 4-simplex lattice which is a fractal lattice embedded in three dimensional space and whose graph is consequently nonplanar. Fractal lattices are constructed iteratively, which makes them suitable for exact recursive treatment if the ramification number is low. Recursive enumerative method on these lattices can provide exact solutions for the close-packed dimer model, in addition to solutions obtained on translationally invariant planar lattices, which proved to be analytically tractable in that case. However, there are no exact solutions of the closepacked dimer model on three dimensional translationally invariant lattices, and result obtained on 4-simplex fractal lattice in this paper, together with the results on other fractal lattices embedded in three dimensional space [22, 24], can give valuable insights into the problem essence.

The paper is organized as follows. In section 2 we shortly describe the closed-

packed dimer model and fractal lattices relevant for this paper. In section 3 we develop recursive method for the enumeration of all dimer configurations on MR family for scaling parameter $2 \leq p \leq 8$ and analyze the model to obtain the entropy. In the same section, we also apply the method on 4-simplex lattice. Discussion of the results and comparison with other lattices are given in section 4 . 


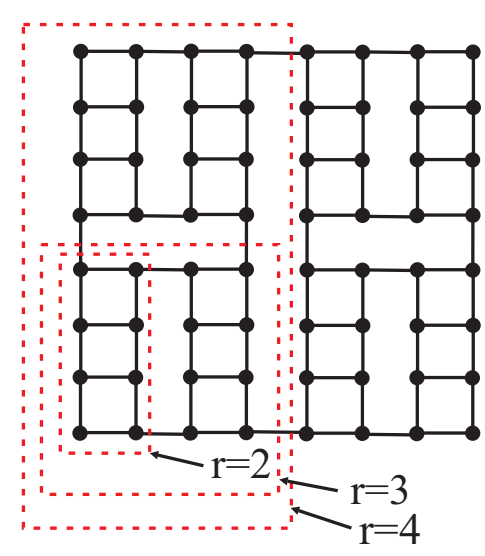

(a) $\mathrm{p}=2, \mathrm{r}=5$

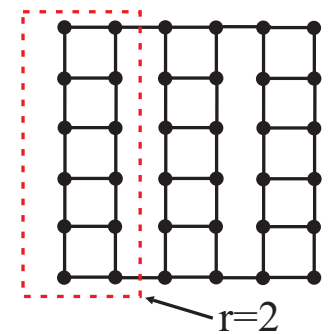

(b) $\mathrm{p}=3, \mathrm{r}=3$

Figure 1. (a) Generator of order $r=5$ for MR lattice with $p=2$. Red dashed rectangles highlight generators of order $r=2, r=3$, and $r=4$ obtained in the subsequent stages of construction. (b) Generator of order $r=3$ for $p=3 \mathrm{MR}$ fractal, with smaller sub-generator (of order $r=2$ ) highlighted by red dashed rectangle. In both cases generator of order $r=1$ (initiator) is a unit square.

\section{The model and lattices}

We assume that dimer is a diatomic molecule, i.e. two monomer units bonded chemically. On a lattice it covers two adjacent lattice points. In close-packed dimer model each lattice site is occupied exactly once by a monomer, and each monomer is connected by a lattice bond with an adjacent monomer into a dimer. Neglecting any other interactions besides hard-core repulsion, partition function of this model is simply the total number of dimer configurations, whose logarithm determines the entropy. Lattices under consideration are MR family of fractals and 4-simplex lattice. Fractals from MR family are labeled with the scaling parameter $p$ (an integer, $2 \leq p \leq \infty$ ). In iterative constructive procedure, structure obtained in the construction step $r$ is called $r$-th order generator and denoted by $G_{r}$. For each particular $p$, at the first step of construction $(r=1)$ one has a graph consisting of four points forming a unit square. Then, $p$ unit squares are joined into the rectangle to obtain the generator of the second order. In the next step, $p$ rectangles are joined into the square, and the process should be repeated infinitely many times to obtain fractal lattice. In figure 1(a) generator of order $r=5$ for $p=2 \mathrm{MR}$ lattice is shown, while in figure $1(b)$ generator of order $r=3$ for $p=3$ member of MR family is shown. Generator of order $r$ for each fractal contains $N_{r}=4 p^{r-1}$ lattice sites (it is also the number of monomers - twice the number of dimers, because of closepacking) and $N_{b r}=\frac{3}{2} N_{r}-2=\frac{6}{p} p^{r}-2$ lattice bonds (edges). Fractal dimension is $d_{f}=\frac{\ln p^{2}}{\ln p}=2$ for each fractal from the family.

Initiator of 4-simplex lattice graph is a complete graph of four points. To obtain second order generator, four initiators are joined into two times larger structure, as shown in figure 2, and the process should be repeated ad infinitum to obtain graph of 


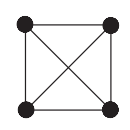

$\mathrm{r}=1$

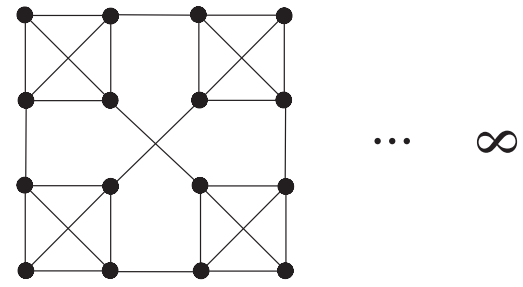

$\mathrm{r}=2$

Figure 2. First two steps of the iterative construction of 4-simplex lattice. Fractal lattice is obtained in the limit $r \rightarrow \infty$.

4-simplex lattice. The number of lattice points in the $r$-th order generator is $N_{r}=4^{r}$, whereas the number of lattice bonds is $N_{b r}=\frac{4}{2} N_{r}-2=2 \cdot 4^{r}-2$. Fractal dimension of the lattice is $d_{f}=\frac{\ln 4}{\ln 2}=2$.

It should be emphasized that for all considered lattices the number of lattice points in generators of any order is even, which is a necessary condition for a lattice to have close-packed dimer covering.

\section{Recursive enumerative method for dimer coverings on fractal lattices}

In this section we will develop the method for recursive enumeration of dimer configurations on aforementioned fractal lattices. Firstly, we establish the exact set of recurrent equations on MR lattices with $2 \leq p \leq 8$ and analyze equations in order to determine the asymptotic form for the numbers of dimer coverings. Also, we numerically find the corresponding entropies per dimer in the thermodynamic limit. Although for some lattice models it was possible to find exact set of recurrence equations on the whole MR family [25], in this case we were not able to do so for the reason that would be explained in the appendix A. Secondly, we apply the same method on 4-simplex lattice and determine the entropy.

\subsection{Dimer coverings on $M R$ fractals}

One close-packed dimer configuration on the 5-th order generator of $p=2 \mathrm{MR}$ lattice is shown in figure 3. In order to develop recurrence equation for the number of dimer coverings on MR lattice, we focus on the corner monomers of smaller generators, that is $G_{4}, G_{3}, G_{2}$, and $G_{1}$, as sub-generators of $G_{5}$ depicted in figure 3. One can notice that the corner monomers of generators of any order form dimers either by the monomers on the same generator or the neighboring ones. We designate the corner monomers as black if their partner is on the same generator, and white if it is on the neighboring one. All generators have four corner monomers and, due to the parity, the only possible combinations are to have all four black, two black and two white, and all four white. Configurations with two black and two white along the diagonals are not possible on MR lattices of any $p$. According to the pairing of the corner monomers, for MR lattices 


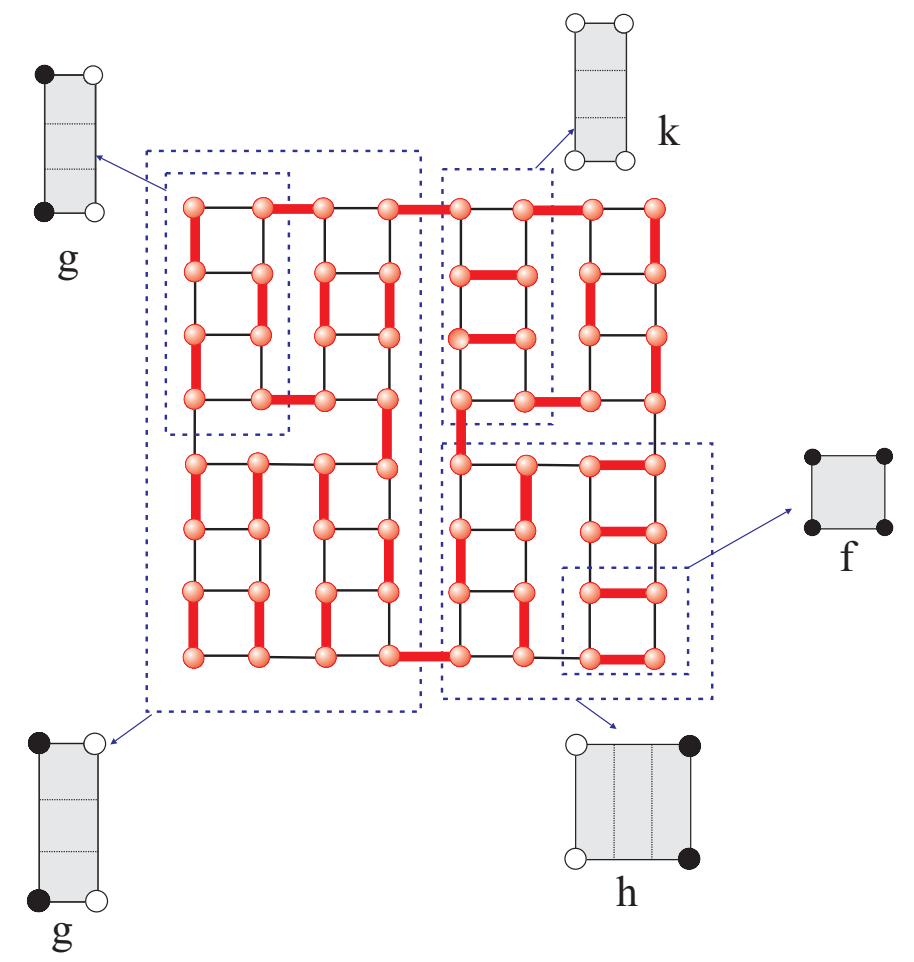

Figure 3. One close-packed dimer configuration on generator $G_{5}$ of $p=2 \mathrm{MR}$ lattice. Dimer configurations on some sub-generators (rectangles or squares) are enclosed into blue dashed rectangles (squares), outlined on the sides and labeled as $f, g, h$ or $k$, depending on the type of the configuration.

of arbitrary $p$, we introduce four types of configurations on generators of any order $r$, namely $f, g, h$ and $k$ with the following meaning:

- $f$ - denotes each dimer configuration in which all four corner monomers are black. They form dimers with the 'internal' monomers, i.e. monomers on the same generator,

- $g$ - denotes each dimer configuration in which two corner monomers are black and belong to the different sub-generators of order $(r-1)$. These two black corner monomers form dimers with the monomers on the same $G_{r}$, while the other two white corner monomers form dimers with the monomers on the two neighboring $G_{r}$,

- $h$ - denotes each dimer configuration in which two corner monomers are black and belong to the same sub-generator of order $(r-1)$. As in type $g$, these two black corner monomers form dimers with the monomers on the same $G_{r}$, while the other two white corner monomers form dimers with the monomers on the two neighboring $G_{r}$,

- $k$ - denotes each dimer configuration in which all four corner monomers are white. They form dimers with the 'external' monomers, i.e. monomers on the neighboring generators. 


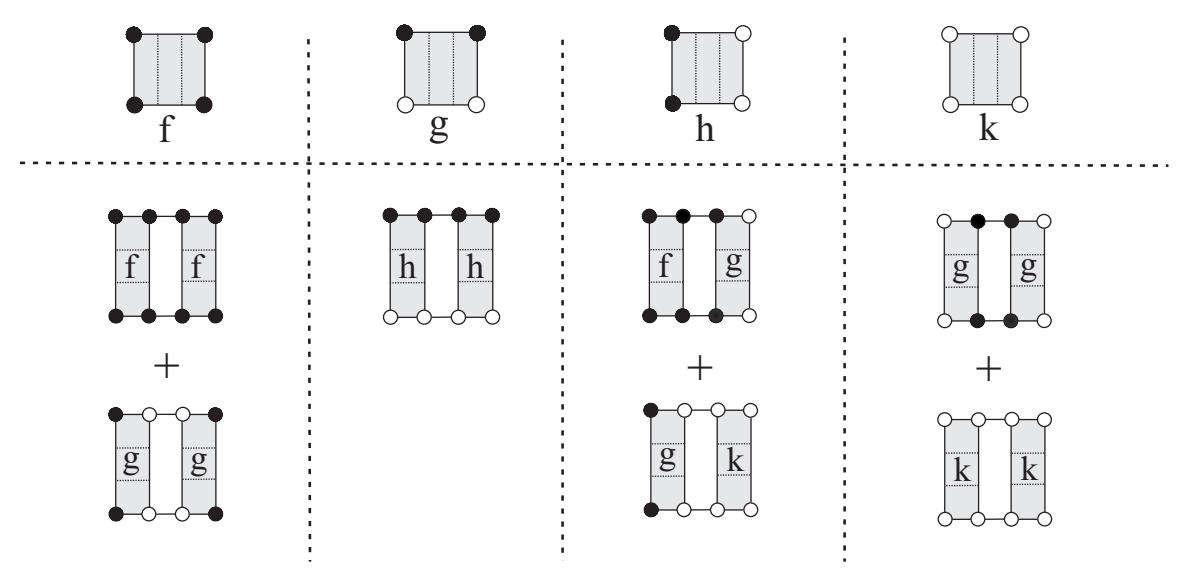

Figure 4. All types of dimer configurations on arbitrary generator $G_{r+1}$ of $p=2 \mathrm{MR}$ fractal, denoted as $f, g, h$ and $k$, and their composing configurations on generators $G_{r}$, from which recurrence equations (3.1) stem.

In figure 3, all four types of configurations are enframed and schematically represented on the sides. In this schematic representation only corner monomers are shown with the internal structure of generators omitted, except that the two consecutive subgenerators are indicated by the dashed lines in order to easily distinguish between $g$ and $h$ configurations.

The numbers of configurations of each type on the $r$-th order generator are designated as $f_{r}, g_{r}, h_{r}$ and $k_{r}$. The total number of dimer configurations on $G_{r}$ is given by $f_{r}$, and can be determined through the system of recurrence equations that involve all four configurations. The closed system of the recurrence equations for $p=2$ is given as

$$
\begin{aligned}
& f_{r+1}=f_{r}^{2}+g_{r}^{2}, \\
& g_{r+1}=h_{r}^{2}, \\
& h_{r+1}=f_{r} g_{r}+g_{r} k_{r}, \\
& k_{r+1}=g_{r}^{2}+k_{r}^{2},
\end{aligned}
$$

and can be inferred on the basis of figure 4, where we illustrate how each configuration on the $(r+1)$-th order generator can be composed from the configurations on the two constituent $r$-th order generators. Similarly, with the help of figure 5, one can formulate recurrence equations for $p=3$ as

$$
\begin{aligned}
& f_{r+1}=f_{r}^{3}+2 f_{r} g_{r}^{2}+g_{r}^{2} k_{r}, \\
& g_{r+1}=h_{r}^{3}, \\
& h_{r+1}=f_{r}^{2} g_{r}+f_{r} g_{r} k_{r}+g_{r}^{3}+g_{r} k_{r}^{2}, \\
& k_{r+1}=f_{r} g_{r}^{2}+2 g_{r}^{2} k_{r}+k_{r}^{3} .
\end{aligned}
$$

Recurrence equations on fractals with $4 \leq p \leq 8$ are given in appendix A. The initial conditions of these equations are the numbers of configurations on the unit square, and, for each $p$ they are given by: $f_{1}=2$ (both two possible), $g_{1}=1$ (only one of the 

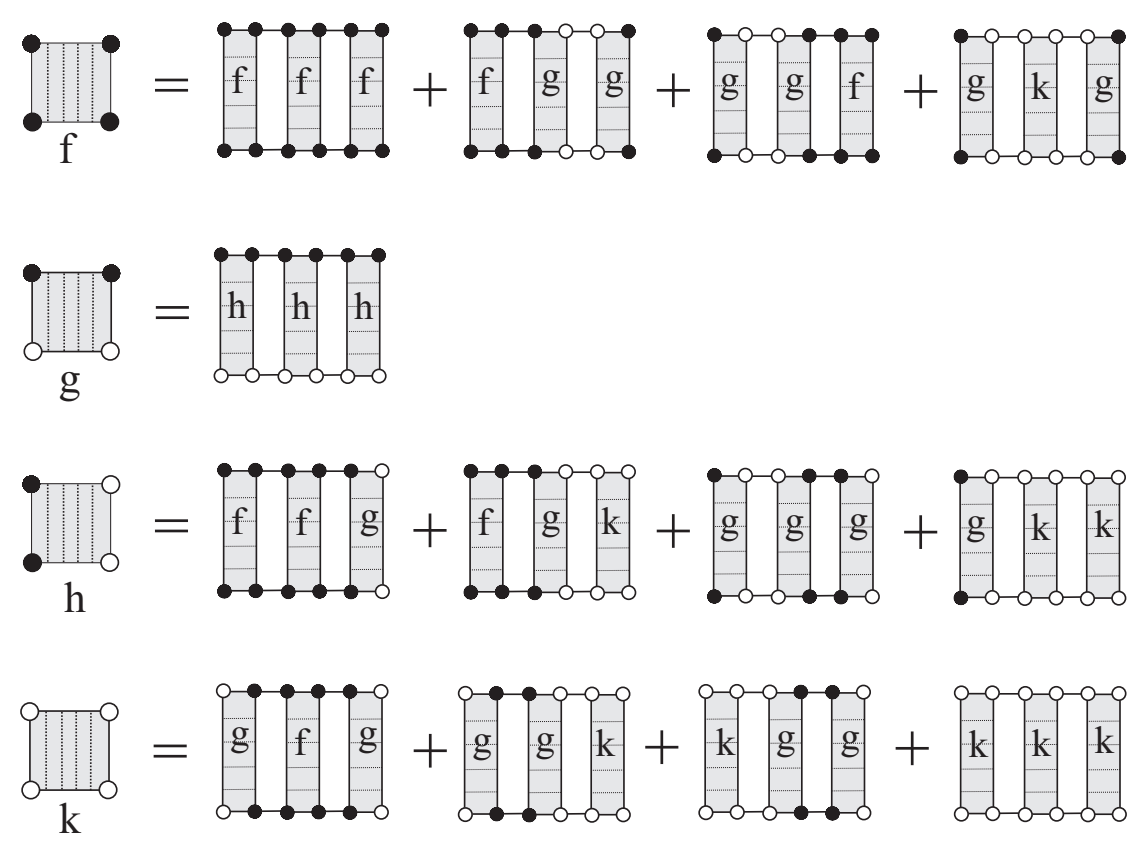

Figure 5. Configurations $f, g, h$, and $k$ on generator $G_{r+1}$ of $p=3$ member of MR fractals and their composing parts on generators $G_{r}$.

Table 1. The numbers of closed-packed dimers on the first five generators for MR fractals labeled by $p=2$ and $p=3$.

\begin{tabular}{|c|ccccc|}
\hline & $r=1$ & $r=2$ & $r=3$ & $r=4$ & $r=5$ \\
\hline$p=2$ & 2 & 5 & 26 & 757 & 575450 \\
$p=3$ & 2 & 13 & 2228 & 12266667328 & 1845787045627790291334622871552 \\
\hline
\end{tabular}

two possible), $h_{1}=1$ (only one of the two possible) and $k_{1}=1$ (the only possible). By computer iteration of recursion relations (3.1), and similarly (3.2), starting with the initial conditions, it is possible to obtain exact numbers of dimer configurations on generators of arbitrary order. These numbers grow very fast with the order $r$ of generator, and for illustration, in table 1 we give the numbers of close-packed dimer configurations on generators of order from $r=1$ to $r=5$ for MR fractals with $p=2$ and $p=3$. Since the systems of difference equations given by (3.1) and (3.2) are not solvable exactly, it is not possible to find exact expressions for $f_{r}$ as functions of $r$. Therefore, we analyze relations numerically and find asymptotic solutions. To make numerical analysis more tractable, we introduce new, rescaled variables defined as $x_{r}=g_{r} / f_{r}, y_{r}=h_{r} / f_{r}$ and $z_{r}=k_{r} / f_{r}$, whose recurrence equations can be obtained from their definitions and the equations (3.1) and (3.2). New equations are

$$
\begin{aligned}
& f_{r+1}=f_{r}^{2}\left(1+x_{r}^{2}\right), \\
& x_{r+1}=\frac{y_{r}^{2}}{1+x_{r}^{2}},
\end{aligned}
$$


Table 2. Entropies per dimer $s_{d}$ of close-packed dimer model on MR fractals with $2 \leq p \leq 8$. The last digits are rounded off.

\begin{tabular}{c|cccc}
\hline$p$ & 2 & 3 & 4 & 5 \\
\hline$s_{d}$ & 0.414750739 & 0.430188671 & 0.441389262 & 0.449006803 \\
\hline$p$ & 6 & 7 & 8 & - \\
\hline$s_{d}$ & 0.454290896 & 0.458114819 & 0.460997823 & - \\
\hline
\end{tabular}

$$
\begin{aligned}
& y_{r+1}=\frac{x_{r}\left(1+z_{r}\right)}{1+x_{r}^{2}}, \\
& y_{r+1}=\frac{x_{r}^{2}+z_{r}^{2}}{1+x_{r}^{2}}
\end{aligned}
$$

for $p=2$, and

$$
\begin{aligned}
& f_{r+1}=f_{r}^{3}\left(1+2 x_{r}^{2}+x_{r}^{2} z_{r}\right), \\
& x_{r+1}=\frac{y_{r}^{3}}{1+2 x_{r}^{2}+x_{r}^{2} z_{r}}, \\
& y_{r+1}=\frac{x_{r}\left(1+z_{r}+x_{r}^{2}+z_{r}^{2}\right)}{1+2 x_{r}^{2}+x_{r}^{2} z_{r}}, \\
& y_{r+1}=\frac{x_{r}^{2}+2 x_{r}^{2} z_{r}+z_{r}^{3}}{1+2 x_{r}^{2}+x_{r}^{2} z_{r}},
\end{aligned}
$$

for $p=3$. Initial values of new variables are $x_{1}=y_{1}=z_{1}=\frac{1}{2}$.

For arbitrary $p$, the recurrence equation for the number $f_{r}$ of close-packed dimers as a function of rescaled variables can be written as

$$
f_{r+1}=f_{r}^{p}\left(1+\sum_{i} a_{i} x_{i}^{\alpha_{i}} z_{i}^{\beta_{i}}\right),
$$

where the coefficients $a_{i}$ and the exponents $\alpha_{i}$ and $\beta_{i}$ depend on $p$. First equation in systems (3.3) and (3.4) are indeed of the given form. Iterating sequences $x, y$ and $z$, we find that for all considered $2 \leq p \leq 8$, elements $x_{r}, y_{r}$ and $z_{r}$ tend to zero very quickly with each iteration step $r$, implying that the equation (3.5) for $r \gg 1$ has the form

$$
f_{r+1} \sim f_{r}^{p} .
$$

This further implies that $f_{r}$ asymptotically grows exponentially with $p^{r}$ i.e. $f_{r} \sim$ $[\text { const }]^{p^{r}}$. Since the number of monomers in $G_{r}$ is given by the $N_{r}=\frac{4}{p} p^{r}$ it follows that $f_{r}$ exponentially grows with the number of monomers

$$
f_{r} \sim \omega^{N_{r}},
$$

for $r \gg 1$. Growth constant $\omega$ is defined through the relation $\ln \omega=\lim _{N_{r} \rightarrow \infty} \frac{\ln f_{r}}{N_{r}}$. To determine growth constant, we take logarithm of the equation (3.5) and divide obtained equation with $N_{r+1}=4 p^{r}$, after which we obtain

$$
\frac{\ln f_{r+1}}{N_{r+1}}=\frac{\ln f_{r}}{N_{r}}+\frac{1}{4 p^{r}} \ln \left(1+\sum_{i} a_{i} x_{i}^{\alpha_{i}} z_{i}^{\beta_{i}}\right) \text {. }
$$




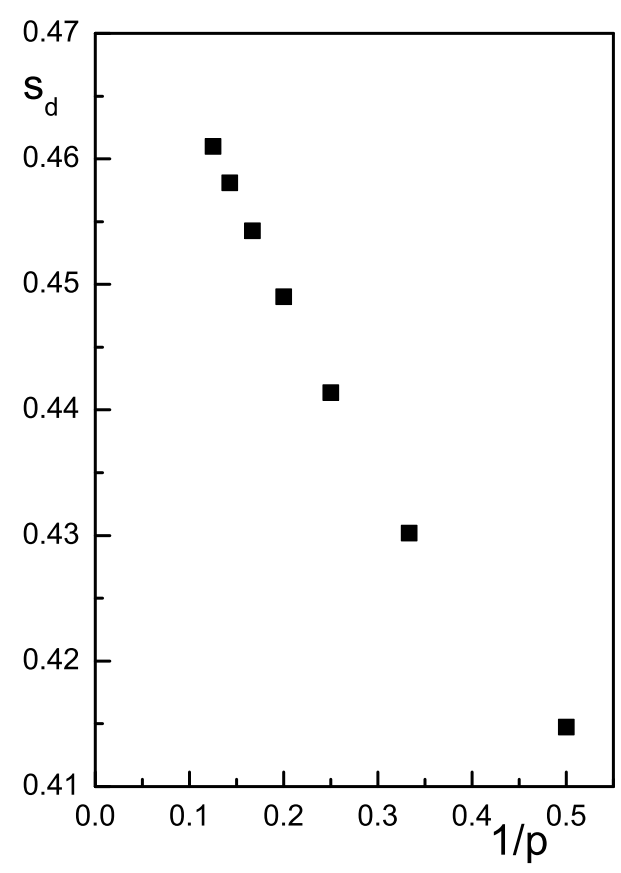

Figure 6. Entropies per dimer of the close-packed dimer model on MR lattices with $2 \leq p \leq 8$ as functions of $1 / p$.

This equation recursively defines sequence of numbers with the elements given by $s_{r}=\frac{\ln f_{r}}{N_{r}}$, so that $s_{m}=\lim _{r \rightarrow \infty} s_{r}=\ln \omega$ holds. For each $p$, the sequence converges very fast, so that for example for $p=3$, after only nine iterations more than twenty significant figures can be achieved, and for higher $p$ convergence is even faster. By numerical iteration, value of $s_{m}$ is determined for each $2 \leq p \leq 8$. Limiting values $s_{m}$ are actually the entropies per monomer in the thermodynamic limit, as can be seen from the definition of the entropy through the number of dimer configurations $S=k_{B} \ln f_{r}$ and equation (3.7). Setting $k_{B}=1$, it follows that $\lim _{N_{r} \rightarrow \infty} S / N_{r}=\ln \omega=s_{m}$. The number of dimers is just half of the number of monomers $N_{r}$, so that the entropy per dimer $s_{d}$ is twice the entropy per monomer. In table 2 we present entropies per dimer in the thermodynamic limit, calculated numerically from the numbers of dimer configurations on MR fractals with $2 \leq p \leq 8$. Entropies $s_{d}$ as functions of $1 / p$ are also presented graphically in figure 6 .

\subsection{Dimer coverings on 4-simplex lattice}

Recursive enumeration of dimer configurations on 4-simplex lattice can be done in a similar manner as on MR lattices. In figure 7 one close-packed dimer configuration on the third order generator of 4-simplex lattice is presented. Again, corner monomers can be all four black - corresponding to $f$ configuration, two black and two white corresponding to $g$ configuration and all four white - corresponding to $h$ configuration. All four corner vertices of 4 -simplex lattice are permutationally equivalent, so it is 


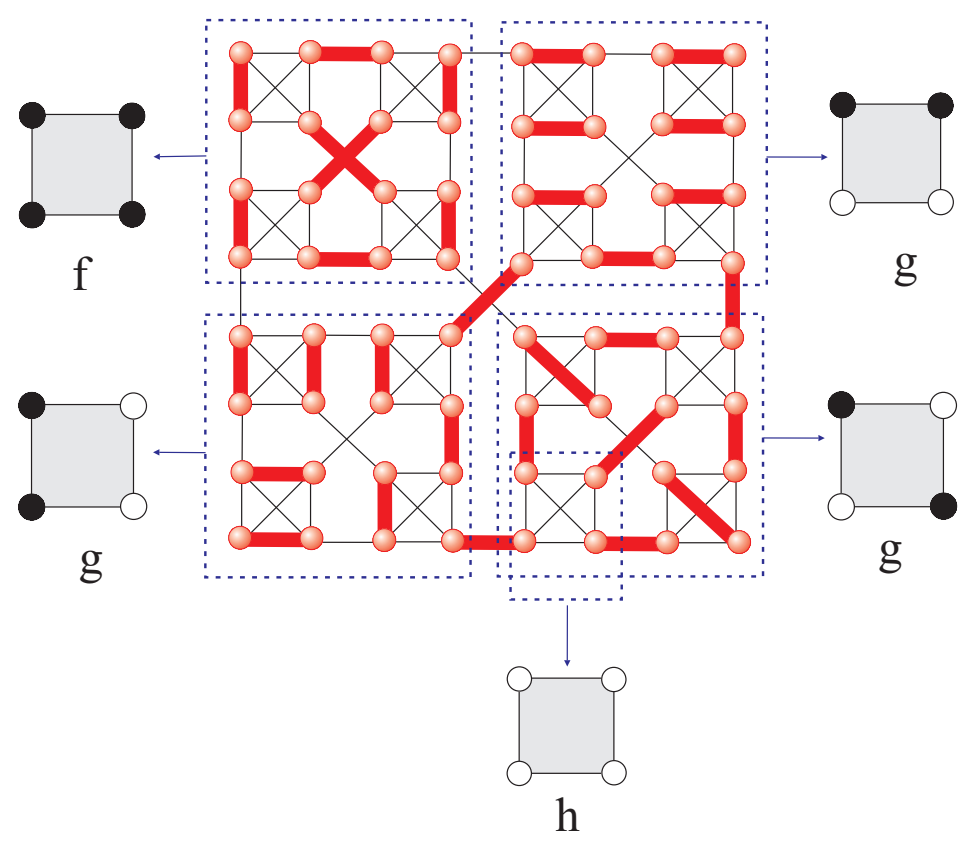

Figure 7. Close-packed dimer configuration on the generator $G_{3}$ of 4-simplex lattice. Possible types of configurations on sub-generators are enframed and schematically shown on the sides.

irrelevant which two monomers are black (white). Four constitutive sub-generators of $G_{3}$ in figure 7 are enframed, with the configurations classified according to the type they belong to, and schematically represented on the sides. Also, one configuration of type $h$ on $G_{1}$ is enframed, and schematically represented on the side. Recurrence equations for all three configurations, as illustrated in figure 8, are given as

$$
\begin{aligned}
& f_{r+1}=f_{r}^{4}+4 f_{r} g_{r}^{3}+3 g_{r}^{4}, \\
& g_{r+1}=f_{r}^{2} g_{r}^{2}+2 f_{r} g_{r}^{3}+g_{r}^{2} h_{r}^{2}+2 g_{r}^{3} h_{r}+2 g_{r}^{4}, \\
& h_{r+1}=h_{r}^{4}+4 g_{r}^{3} h_{r}+3 g_{r}^{4},
\end{aligned}
$$

with the initial conditions $f_{1}=3$ (all three possible), $g_{1}=1$ (one of six possible) and $h_{1}=1$ (the only possible). Analysis of recurrence equations (3.9) proceeds in a similar way as on MR lattices, and we find that the number of all close-packed dimer configurations grows with the number of monomers as

$$
f_{r} \sim \omega^{N_{r}},
$$

with the entropy per dimer $s_{d}=2 \ln \omega=0.571832556$.

\section{Discussion and conclusions}

We have studied the close-packed dimer model on two types of fractal lattices, namely, lattices from MR family with the scaling parameter $2 \leq p \leq 8$ embedded in two dimensional space, and 4 -simplex lattice embedded in three dimensional space. The asymptotic forms for the number of dimer configurations on these lattices have been 

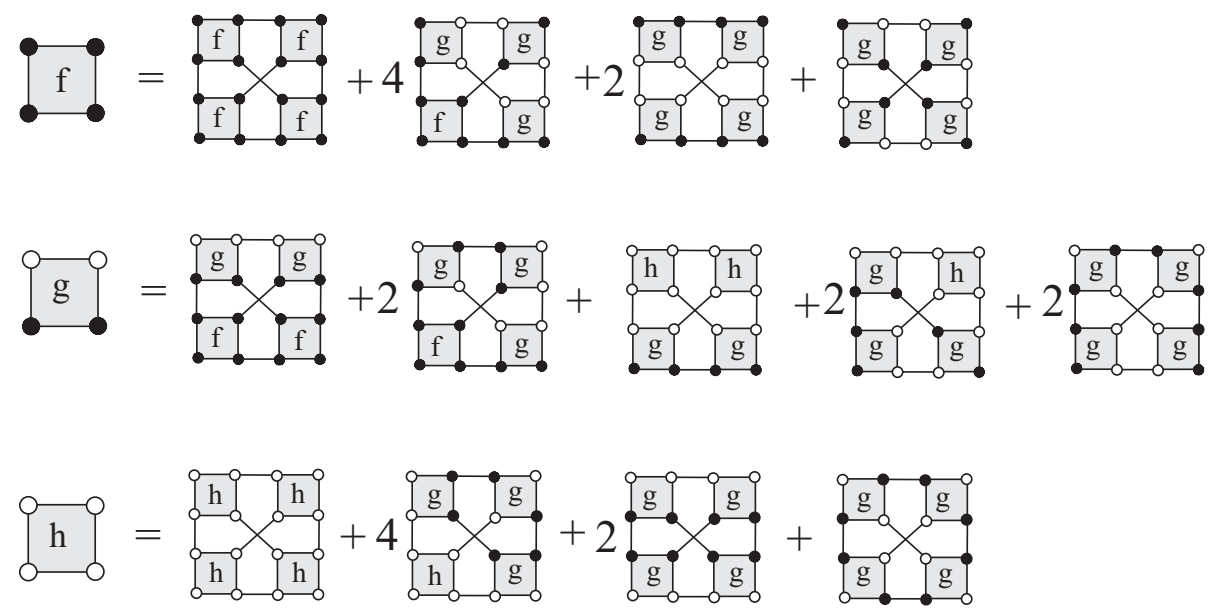

Figure 8. Configurations $f, g$ and $h$ on generator $G_{r+1}$ of 4 -simplex lattice and their composing parts on generators $G_{r}$, which determine corresponding terms in equations (3.9). Multiplication by the factors 4 or 2 are due to the symmetrically related configurations.

determined. It is found that, on all considered fractals, the asymptotic form for number of dimer configurations is a pure exponential function of the number of monomers. In addition, microcanonical entropies per dimer in the thermodynamic limit are determined numerically. On MR lattices, entropy is an increasing function of fractal parameter $p$, as can be seen in table 2. This deserves some insight into the geometry of the lattices. All lattices from this family are constructed iteratively through succession of generators, and all have the same fractal dimension. The ramification number of each lattice is two, and for all latices coordination number of each vertex is three (vertex degree), except for the corner vertices of the largest generator. But, the number of bonds and their distribution are different, and lattices with higher value of $p$ at each construction stage have larger number of bonds (caused by the larger number of vertices), so the configurational space is larger. In figure 6 entropies are shown as functions of inverse scaling parameter $p$, and an obvious question arises: is this sequence convergent when $p \rightarrow \infty$, and if so, what is the limit? We expect that there is a finite limiting value, and suppose that it is smaller than the value of entropy $s_{s q}=0.583121808$ obtained on the square lattice in [2]. This conclusion is justified by the fact that all MR lattices resemble to the square lattice since they can be obtained from it by deleting some bonds. Consequently, MR lattices have smaller number of bonds than the square lattice of equal size.

On the other hand, entropy obtained on 4-simplex lattice is $s_{\text {sim }}=0.571832556$, and compared with the values in table 2, we see that it is larger than any value on MR fractals considered. Coordination number of 4-simplex lattice is four and thus greater than for MR lattices, but for MR fractals with $p \geq 5$ at $r$-th construction stage there are more bonds than on 4-simplex lattice at the same stage. However, MR lattices are highly anisotropic and their connectedness does not allow some configurations (in subsection 3.1 configurations with two black and two white along the square (rectangle) 
diagonals were forbidden), resulting in smaller entropy. Value $s_{\text {sim }}$ obtained here, should also be compared with the value of entropy of the same model studied on the Sierpinski gasket (SG) embedded in three dimensional space [22], which is $s_{3 d S G}=0.857927798$, thus much larger than $s_{\text {sim }}$. These two lattices both have tetrahedral structure, but vertices of the neighboring tetrahedra are glued on $3 d \mathrm{SG}$, so that the coordination number of this lattice is six. Furthermore, entropy per dimer is larger on square lattice than on 4-simplex lattice, although they both have the same coordination number.

To conclude, we can confirm that besides the coordination number, which is the most relevant lattice parameter that determines the number of close-packed dimer configurations, other lattice parameters and geometric constraints are important too. In favor of latter is the finding that on translationally invariant lattices the boundary effects play an important role. For example, on square lattice the entropy is the same for both open and periodic boundary conditions [2,6], whereas on hexagonal lattice it strongly depends on the boundary [17,26]. Extensions of the studies to account for cylindrical boundary conditions have also been done [27,28]. Similar problem has been encountered on other 'close-packed' models, namely Hamiltonian walk problem, where entropic exponent $\gamma$ depended on the boundary conditions [29]. With respect to all these unresolved questions, we can say that additional studies should be conducted in order to specify all relevant parameters on fractal lattices as well as on translationally invariant ones, taking into account all metric properties of lattices.

Finally, we would like to mention that the dimer model considered here could be supplemented with the interaction weights and studied on fractal lattices. Interacting dimer model is extensive and physically more interesting, but also more difficult to approach.

\section{Acknowledgments}

This paper has been done as a part of the work within the project No. 171015, funded by the Ministry of Education, Science and Technological Development of the Republic of Serbia.

\section{Appendix A.}

In this appendix we give recurrence equations for the determination of the number of dimer coverings on MR fractals with $4 \leq p \leq 8$. We explicitly state the recurrence equations only for the variables $f$ and $h$, since the recurrence relation for the configuration $g$ is simply $g_{r+1}=h_{r}^{p}$ for each $p$, and for $k$ it could be obtained from the recurrence equation for $f$ with the substitution $k \leftrightarrow f$ on both sides of equation.

$$
\begin{aligned}
\quad & =4: \\
f_{r+1} & =f_{r}^{4}+3 f_{r}^{2} g_{r}^{2}+2 f_{r} g_{r}^{2} k_{r}+g_{r}^{4}+g_{r}^{2} k_{r}^{2}, \\
h_{r+1} & =f_{r}^{3} g_{r}+f_{r}^{2} g_{r} k_{r}+2 f_{r} g_{r}^{3}+f_{r} g_{r} k_{r}^{2}+2 g_{r}^{3} k_{r}+g_{r} k_{r}^{3} .
\end{aligned}
$$




$$
\begin{aligned}
p & =5: \\
f_{r+1} & =f_{r}^{5}+4 f_{r}^{3} g_{r}^{2}+3 f_{r}^{2} g_{r}^{2} k_{r}+3 f_{r} g_{r}^{4}+2 f_{r} g_{r}^{2} k_{r}^{2}+2 g_{r}^{4} k_{r}+g_{r}^{2} k_{r}^{3}, \\
h_{r+1} & =f_{r}^{4} g_{r}+f_{r}^{3} g_{r} k_{r}+3 f_{r}^{2} g_{r}^{3}+f_{r}^{2} g_{r} k_{r}^{2}+4 f_{r} g_{r}^{3} k_{r}+g_{r}^{5}+f_{r} g_{r} k_{r}^{3}+3 g_{r}^{3} k_{r}^{2}+g_{r} k_{r}^{4} .
\end{aligned}
$$

$p=6$ :

$$
\begin{aligned}
f_{r+1} & =f_{r}^{6}+5 f_{r}^{4} g_{r}^{2}+4 f_{r}^{3} g_{r}^{2} k_{r}+6 f_{r}^{2} g_{r}^{4}+3 f_{r}^{2} g_{r}^{2} k_{r}^{2}+6 f_{r} g_{r}^{4} k_{r}+g_{r}^{6}+2 f_{r} g_{r}^{2} k_{r}^{3}+3 g_{r}^{4} k_{r}^{2} \\
& +g_{r}^{2} k_{r}^{4}, \\
h_{r+1} & =f_{r}^{5} g_{r}+f_{r}^{4} g_{r} k_{r}+4 f_{r}^{3} g_{r}^{3}+f_{r}^{3} g_{r} k_{r}^{2}+6 f_{r}^{2} g_{r}^{3} k_{r}+3 f_{r} g_{r}^{5}+f_{r}^{2} g_{r} k_{r}^{3}+6 f_{r} g_{r}^{3} k_{r}^{2} \\
& +3 g_{r}^{5} k_{r}+f_{r} g_{r} k_{r}^{4}+4 g_{r}^{3} k_{r}^{3}+g_{r} k_{r}^{5} .
\end{aligned}
$$

$p=7$ :

$$
\begin{aligned}
f_{r+1} & =f_{r}^{7}+6 f_{r}^{5} g_{r}^{2}+5 f_{r}^{4} g_{r}^{2} k_{r}+10 f_{r}^{3} g_{r}^{4}+4 f_{r}^{3} g_{r}^{2} k_{r}^{2}+12 f_{r}^{2} g_{r}^{4} k_{r}+4 f_{r} g_{r}^{6}+3 f_{r}^{2} g_{r}^{2} k_{r}^{3} \\
& +9 f_{r} g_{r}^{4} k_{r}^{2}+3 g_{r}^{6} k_{r}+2 f_{r} g_{r}^{2} k_{r}^{4}+4 g_{r}^{4} k_{r}^{3}+g_{r}^{2} k_{r}^{5}, \\
h_{r+1} & =f_{r}^{6} g_{r}+f_{r}^{5} g_{r} k_{r}+5 f_{r}^{4} g_{r}^{3}+f_{r}^{4} g_{r} k_{r}^{2}+8 f_{r}^{3} g_{r}^{3} k_{r}+6 f_{r}^{2} g_{r}^{5}+f_{r}^{3} g_{r} k_{r}^{3}+9 f_{r}^{2} g_{r}^{3} k_{r}^{2} \\
& +9 f_{r} g_{r}^{5} k_{r}+f_{r}^{2} g_{r} k_{r}^{4}+8 f_{r} g_{r}^{3} k_{r}^{3}+g_{r}^{7}+6 g_{r}^{5} k_{r}^{2}+f_{r} g_{r} k_{r}^{5}+5 g_{r}^{3} k_{r}^{4}+g_{r} k_{r}^{6} .
\end{aligned}
$$

$$
p=8:
$$

$$
\begin{aligned}
f_{r+1} & =f_{r}^{8}+7 f_{r}^{6} g_{r}^{2}+6 f_{r}^{5} g_{r}^{2} k_{r}+15 f_{r}^{4} g_{r}^{4}+5 f_{r}^{4} g_{r}^{2} k_{r}^{2}+20 f_{r}^{3} g_{r}^{4} k_{r}+10 f_{r}^{2} g_{r}^{6}+4 f_{r}^{3} g_{r}^{2} k_{r}^{3} \\
& +18 f_{r}^{2} g_{r}^{4} k_{r}^{2}+12 f_{r} g_{r}^{6} k_{r}+g_{r}^{8}+3 f_{r}^{2} g_{r}^{2} k_{r}^{4}+12 f_{r} g_{r}^{4} k_{r}^{3}+6 g_{r}^{6} k_{r}^{2}+2 f_{r} g_{r}^{2} k_{r}^{5} \\
& +5 g_{r}^{4} k_{r}^{4}+g_{r}^{2} k_{r}^{6} \\
h_{r+1} & =f_{r}^{7} g_{r}+f_{r}^{6} g_{r} k_{r}+6 f_{r}^{5} g_{r}^{3}+f_{r}^{5} g_{r} k_{r}^{2}+10 f_{r}^{4} g_{r}^{3} k_{r}+16 f_{r}^{3} g_{r}^{5}+f_{r}^{4} g_{r} k_{r}^{3}+12 f_{r}^{3} g_{r}^{3} k_{r}^{2} \\
& +16 f_{r}^{2} g_{r}^{5} k_{r}+f_{r}^{3} g_{r} k_{r}^{4}+12 f_{r}^{2} g_{r}^{3} k_{r}^{3}+18 f_{r} g_{r}^{5} k_{r}^{2}+4 g_{r}^{7} k_{r}+f_{r}^{2} g_{r} k_{r}^{5}+10 f_{r} g_{r}^{3} k_{r}^{4} \\
& +10 g_{r}^{5} k_{r}^{3}+f_{r} g_{r} k_{r}^{6}+6 g_{r}^{3} k_{r}^{5}+g_{r} k_{r}^{7} .
\end{aligned}
$$

As one can see from equations (A.1)-(A.5) the number of terms and their coefficients increase rapidly with $p$. Analyzing the model on MR lattices with arbitrary $p$, we could formally write down recurrence equation for $f$ in the following form

$$
f_{r+1}=f_{r}^{p}+\sum_{i=1}^{p-1} \sum_{j=0}^{n_{i}} c_{i j} f^{\alpha_{i j}} g^{\beta_{i j}} k^{\gamma_{p i j}},
$$

where the upper limit of the second sum, the coefficients, and the exponents, all depend on $p$. The first term is obvious, and represents the only one possible way to obtain $f$ configuration on $G_{r+1}$ composed from $f$ configurations on each $G_{r}$, and this configuration 
0

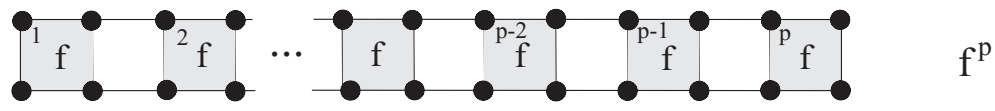

1
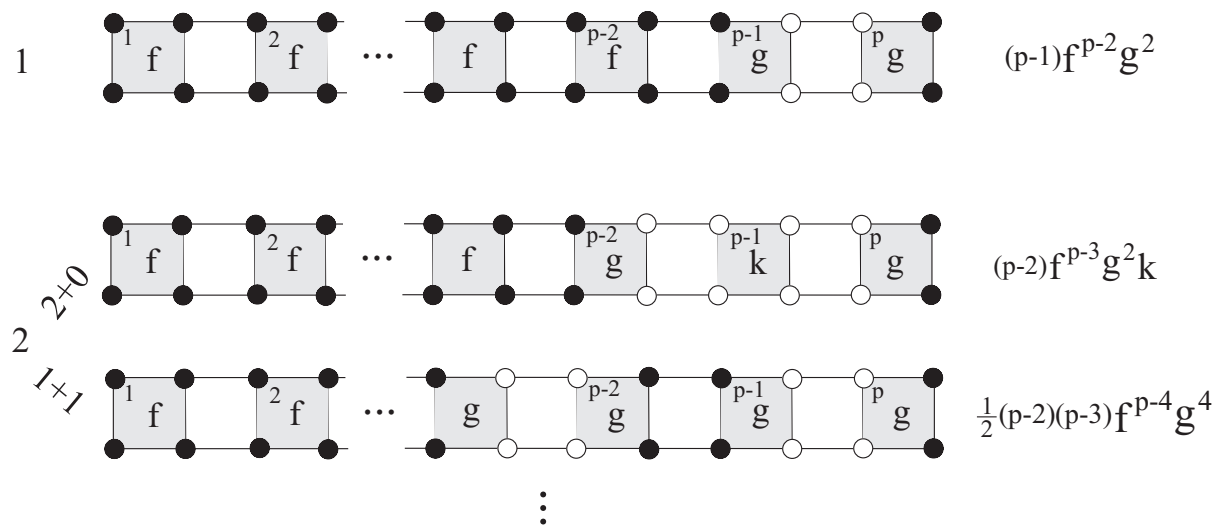

Figure A1. First few terms and their coefficients in recurrence equation (A.6) for the total number of dimers $f_{r}$ on MR lattice of large, arbitrary $p$.

is shown in the first row of figure A1, The double sum in equation (A.6) is nontrivial. The first sum represents terms that correspond to situations in which one, two, three and so on 'empty' rectangles, with all four corner monomers white, can be chosen out of $(p-1)$ such rectangles, and, the second sum distinguishes whether these rectangles are consecutive or not. There are $(p-1)$ ways to choose one 'empty' rectangle, so that coefficient $c_{10}$ of that term in the sum is $(p-1)$, (second row in figure A1). Then, two consecutive rectangles can be chosen in $(p-2)$ ways, which gives $c_{20}=p-2$. Two non-consecutive rectangles can be chosen in $c_{21}=\frac{1}{2}(p-2)(p-3)$ ways (third row in figure A1). Three rectangles can all be consecutive, one separated and two consecutive and all three non-consecutive. Thus, the upper limit of the second sum $n_{i}$ is the number of ways in which a positive integer can be represented as the sum, which in combinatorics is known as partition. There is no simple formulae for the partition function of a positive integer, but it is known that asymptotically it grows exponentially with the square root of its argument. Therefore, the number of terms increases very fast, and even if we succeeded to enumerate and sort the terms in recurrence equations, they would be too cumbersome to analyze. At this point, we would like to notice that other methods might be more efficient in handling the problem on the whole family of fractals, especially in the large $p$ limit.

\section{References}

[1] Fowler R J and Rushbrooke G S 1937 Trans. Faraday. Soc. 331272

[2] Kasteleyn P W 1961 Physica 271209

[3] Kasteleyn P W 1963 J. Math. Phys. 4287

[4] Temperley H N V and Fisher M E 1961 Philos. Magazine 61061

[5] Fisher M E and Temperley H N V 1960 Rev. Mod. Phys. 321029 
[6] Fisher M E 1961 Phys. Rev. 1241664

[7] Kenyon R, Okounov A and Sheffield M E 2006 Ann. Math. 1631019

[8] Fisher M E 1966 J. Math. Phys. 71776

[9] Nienhuis B 1987 Coulomb gas formulation of two-dimensional phase transitions, a chapter in Phase transitions and critical phenomena 11 (Academic Press, London) p54

[10] Kondev J and Henley C L 1995 Phys. Rev. B $\mathbf{5 2} 6628$

[11] Iqbal A, Nekrasov N, Okounkov A and Vafa C 2008 JHEP 0411

[12] Alet F, Jacobsen J L, Misguich G, Pasquier V, Mila F and Troyer M 2005 Phys. Rev. Lett. 94 235702

[13] Alet F, Ikhlef Y, Jacobsen J L, Misguich G and Pasquier V 2006 Phys. Rev. E 74041124

[14] Cohn H, Kenyon R and Propp J 2001 J. Amer. Math. Soc. 14297

[15] Kenyon R W, Propp J G and Wilson D B 2000 Electron. J. Comb. 7 \#R25

[16] Temperley H N V 1974 London Math. Soc. Lecture Notes Series 13202

[17] Elser V 1984 J. Phys. A: Math. Gen. 171509

[18] Fendley P, Moessner R and Sondhi S L 2002 Phys. Rev. B 66214513

[19] Wu F Y 2006 Int. J. Mod. Phys. B 20535

[20] Harris A B and Cohen M 2006 J. Chem. Phys. 125184107

[21] D'angeli D, Dono A and Nagnibeda T 2012 Eur. J. Comb. 331484

[22] Chang S C and Chen L C 2008 J. Stat. Phys. 131631

[23] Dhar D 1977 J. Math. Phys. 18577

[24] Dhar D and Chandra S 2008 Phys. Rev. Lett. 100120602

[25] Marčetić D, Elezović-Hadžić S, Adžić N and Živić I 2019 J. Phys. A 52125001

[26] Grensing D and Grensing G 1983 J. Math. Phys. 24620

[27] Yan W, Yeh Y N and Zhang F 2008 Physica A $\mathbf{3 8 7} 6069$

[28] Li S and Yan W 2016 Physica A 452251

[29] Duplantier B and David F 1988 J. Stat. Phys. 51327 